\title{
Modification of the Optical Performance of Fiber Bragg Gratings Using Femtosecond Laser Micromachining
}

\author{
Hamidreza Alemohammad ${ }^{*}$, Ehsan Toyserkani ${ }^{*}$ \\ * Rapid Prototyping Laboratory, Department of Mechanical and Mechatronics Engineering \\ University of Waterloo, 200 University Ave. West, Waterloo, Ontario, Canada N2L 3G1 \\ E-mail: shalemoh@uwaterloo.ca
}

\begin{abstract}
This paper describes the use of femtosecond laser pulses to selectively modify the optical performance of as-fabricated fiber Bragg gratings (FBG) written in single mode optical fibers. As a result of the irradiation of FBGs by femtosecond laser pulses generated from a Ti:Sapphire amplifier, the resonance Bragg wavelength is changed, or split into two separate individual wavelengths. In addition, the bandwidth of the reflection peak is increased. Further analyses show that a change of $6.09 \times 10^{-4}$ in the refractive index is achieved in a single pass of the laser beam. Periodic microgrooves are also inscribed along the fiber using femtosecond pulses. In addition to the optical performance, the sensing performance of the FBGs is increased by inscription of periodic microgrooves in the cladding of the fiber. The micromachined fibers are used for simultaneous measurement of temperature and concentration of liquids.
\end{abstract}

DOI: $10.2961 / \mathrm{jlmn} .2009 .01 .0012$

Keywords: Femtosecond laser micromachining, fiber Bragg grating, optical performance, simultaneous measurement of temperature and concentration

\section{Introduction}

Fiber Bragg gratings (FBG), which are submicron periodic modulations of the index of refraction in optical fibers, are widely used in sensing and communication industries. Fiber Bragg gratings are usually inscribed in single mode optical fibers using Ultra Violet (UV) radiation using masks [1]. The gratings can be customized by changing the masks which is a time consuming and expensive process.

With the advancements in the femtosecond laser micromachining of transparent materials, e.g., glass, the performance of the as-fabricated FBGs can be selectively modified.

The interaction of femtosecond laser pulses with dielectric materials, such as glass, has been a research topic during the past years [2-5]. The optical fiber is made of silica, which is transparent to visible and near-infrared (NIR) spectra of electromagnetic radiation. However, ultrashort laser pulses, i.e., tenth of picoseconds or femtosecond, at $800 \mathrm{~nm}$ enable both bulk modification and surface micromachining of silica.

Surface material ablation of silica, associated with femtosecond laser radiation involves some fundamental processes. When the material surface is irradiated by a laser pulse, the electrons in the conduction band are excited through multiphoton ionization. The density of the electrons increases through avalanche ionization until the frequency of the formed plasma reaches the frequency of the laser radiation. The high density plasma is strongly absorbent to the laser energy. This energy is transferred from the plasma to the lattice after the laser pulse is gone. This transfer of energy occurs in a time scale much smaller than the thermal diffusion time, and can result in material ablation $[2,5,6]$.

In addition to surface micromachining, structural modifications like change in the refractive index and stress in- duction can be accomplished due to nonlinear phenomena such as photoionization that occur during the interaction of femtosecond laser pulses with silica [7-9]. Also, femtosecond lasers have been used for the induction of birefringence in optical fibers by exposing the cladding to the laser radiation [10] and for direct writing of Bragg gratings in the core of optical fibers for sensing and fiber laser applications [11-14]. The fabrication of intracore Bragg gratings by the lateral illumination of the optical fibers using phase mask scanning technique is presented in [13]; to enhance the sensing performance of FBGs, a femtosecond laser was used for the inscription of high-temperature stable Bragg gratings in polarization maintaining (PM) fibers. The photo-enhanced birefringence caused by the femtosecond pulses resulted in FBG sensors with dual-parameter sensing capability in a wide range [14]. Bragg gratings have also been inscribed in the core of the Yb-doped fibers used in fiber lasers [15].

With the great potentials of femtosecond laser pulses in micromachining of silica, they can be effectively incorporated for customizing as-fabricated FBGs. In this paper, we report the use of femtosecond laser pulses to selectively modify the performance of as-fabricated FBGs. In the experiments, an as-fabricated FBG is radiated by femtosecond laser pulses moving in a specified path relative to the fiber. The reflectivity spectra obtained after laser processing show that the femtosecond laser radiation can effectively modify the reflectivity characteristics of the FBGs. Shift in the Bragg wavelength, splitting the single FBG peak into two individual peaks, and a change in the bandwidth of the Bragg reflection are obtained. In addition, the sensitivity of the FBGs for simultaneous measurement of temperature and concentration of liquids is increased as a result of the inscription of periodic micro-grooves in the cladding of the fiber. 


\section{FBG Theory}

In FBGs, the coupling between the forward and backward propagating modes results in a resonance condition. The resonance condition occurs at the Bragg wavelength $\left(\lambda_{B}\right)$ which is a function of the effective mode index of refraction ( $\left.n_{\text {eff }}\right)$ and the grating pitch ( $\Lambda$ ), as follows [16]:

$$
\lambda_{B}=2 n_{\text {eff }} \Lambda
$$

Any permanent change in the indices of refraction of the core or cladding of optical fiber, induced by external sources such as femtosecond laser radiation, can modify $n_{\text {eff }}$ and the spectral response.

\section{Experimental Setup}

Fig. 1(a) shows a schematic diagram of the femtosecond laser setup and the position of the optical fiber relative to the laser beam. The FBGs were installed on a high precision 3-dimentional motion system manufactured by Aerotech Inc., and were moved across the laser beam to inscribe periodic grooves along the fiber. An NIR integrated, diodepumped, ultrafast Ti:Sapphire regenerative amplifier (Libra) manufactured by Coherent Inc. was used for the experiments. The laser produced light of $800 \mathrm{~nm}$ wavelength in $100 \mathrm{fs}$ pulses with a repetition rate of $1 \mathrm{kHz}$. The maximum pulse energy was $1 \mathrm{~mJ}$ which was attenuated to 20-40 $\mu \mathrm{J}$ for the experiments. A $15 \times$ lens (NA=0.28) was used to focus the beam to the processing zone. The laser spot diameter was $\sim 8 \mu \mathrm{m}$, and the depth of focus (DOF) was $\sim 21$ $\mu \mathrm{m}$. The beam focal point parameters are small relative to the cladding diameter of the fiber $(125 \mu \mathrm{m})$, but in the experiments the laser beam was defocused on the fiber cladding surface to create larger features. The circular shape of the fiber gave non-circular intensity distribution at the process zone. In this case, the beam diameter along the fiber axis ( $\mathrm{z}$ axis) was smaller than that in the direction perpendicular to the fiber axis (x axis) [17].

Fiber Bragg gratings with a central wavelength of around $1550 \mathrm{~nm}$ were used for the experiments. The FBGs were outsourced from a supplier (O/E Land Inc.), and according to the data sheets they were fabricated by UV radiation with appodized grating.

The optical fibers were coated with a protective polymeric layer to protect them during shipping and handling. Since the layer could modify the laser beam characteristics during the beam exposure, the layer was removed by a chemical stripping process before the laser micromachining. To do that, the FBGs were immersed in acetone for 10-15 minutes to dissolve the polymer.

In the experiments, the fiber was moved continuously at a constant speed with a pre-specified pitch to periodically irradiate the fiber, as shown in Fig. 1(b).

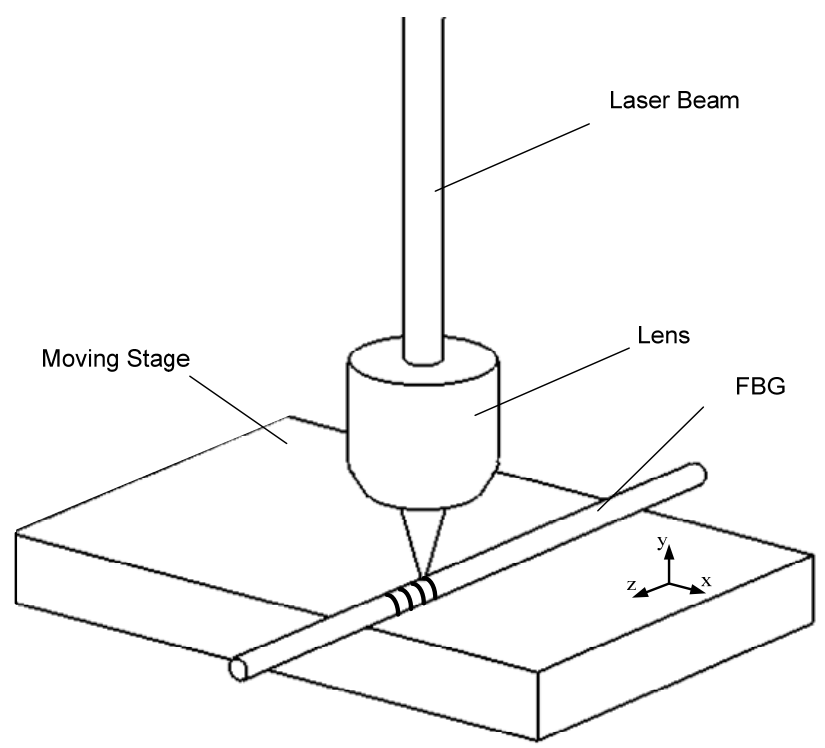

(a)

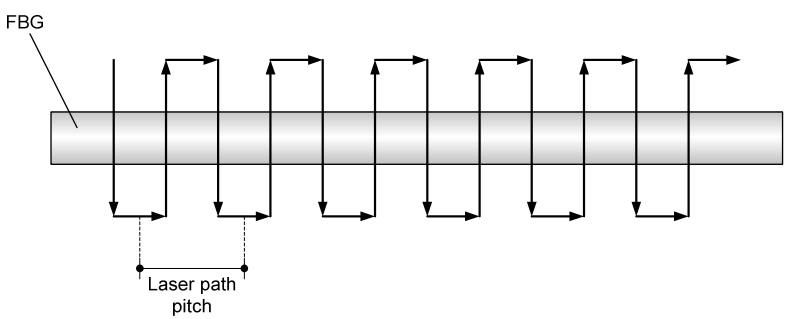

(b)

Fig. 1 (a) Femtosecond laser workstation setup, (b) laser path relative to the fiber

\section{Experimental Results and Discussions: Optical Re- sponse}

\subsection{Shift of Bragg Wavelength}

In the first experiment, the laser beam was attenuated to an average power of $40 \mathrm{~mW}$ (equal to $40 \mu \mathrm{J}$ pulse energy), and the fiber was moved continuously with a pitch of 50 $\mu \mathrm{m}$ and speed of $100 \mu \mathrm{m} / \mathrm{s}$. Laser micromaching was performed on the full length of the grating $(10 \mathrm{~mm})$. The laserfiber distance was set in such a way that the exposure area on the fiber was $22 \mu \mathrm{m}$. This was obtained by measuring the trace width of the irradiated regions. As a result, the pulse intensity was $1.03 \times 10^{14} \mathrm{~W} / \mathrm{cm}^{2}$. The original and final reflection spectra of the FBG are shown in Fig. 2. As seen, the central wavelength of the fiber is shifted by 465 $\mathrm{pm}$. The red shift in the Bragg wavelength is attributed to the change in the fiber index of refraction. As the index of refraction at the locations exposed to the femtosecond laser pulses increases, the effective mode index of refraction increases affecting the reflection spectrum. Using (1), the change in the core index of refraction at the points of laser exposure is $\Delta n \approx 4.33 \times 10^{-4}$. 


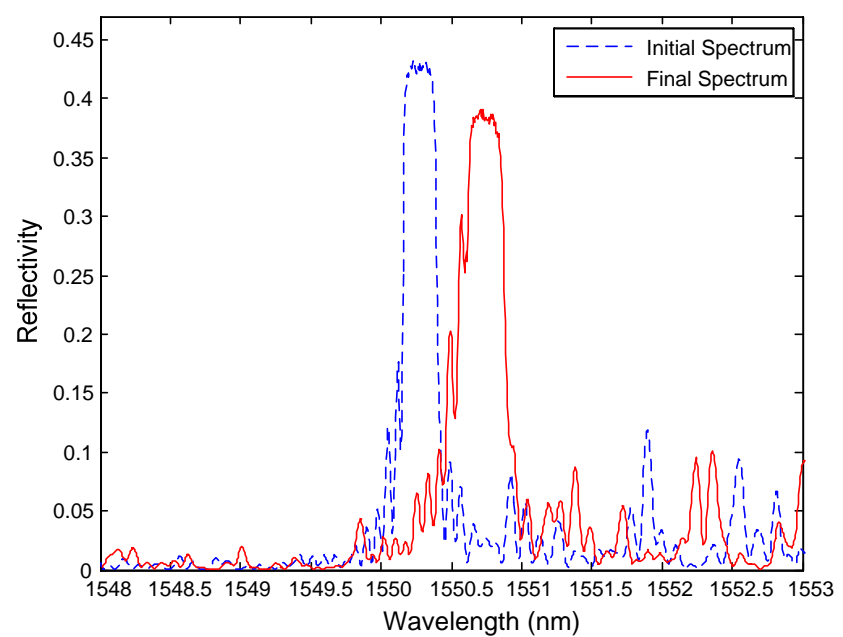

Fig. 2 Initial and micromachined FBGs reflection spectra at a pulse intensity of $1.03 \times 10^{14} \mathrm{~W} / \mathrm{cm}^{2}$

\subsection{Dual Peak Reflectivity}

In another experiment, the laser beam was attenuated to a power of $17.5 \mathrm{~mW}$ (equal to $17.5 \mu \mathrm{J}$ pulse energy). The exposure area in the fiber was $10 \mu \mathrm{m}$ resulting in a pulse intensity of $1.45 \times 10^{14} \mathrm{~W} / \mathrm{cm}^{2}$. The fiber was moved continuously with a pitch of $10 \mu \mathrm{m}$ and speed of $100 \mu \mathrm{m} / \mathrm{s}$ to radiate half of the length of the grating $(5 \mathrm{~mm})$. The reflectivity spectra are shown in Fig. 4. As seen, two resonance wavelengths are generated in FBG at $1550.33 \mathrm{~nm}$ and $1550.99 \mathrm{~nm}$. Accordingly, the change in the index of refraction is $\Delta n \approx 6.09 \times 10^{-4}$.

\subsection{Bandwidth Increase}

Fig. 4 shows the results of an experiment in which the fiber was moved periodically with a pitch of $50 \mu \mathrm{m}$, so half of the length of the grating $(5 \mathrm{~mm})$ was micromachined with a laser power of $40 \mathrm{~mW}$ and continuous laser scanning speed of $100 \mu \mathrm{m} / \mathrm{s}$. The exposure area was $24 \mu \mathrm{m}$ resulting in a pulse intensity of $9.06 \times 10^{13} \mathrm{~W} / \mathrm{cm}^{2}$. As seen, the bandwidth of the reflectivity signal was increased. The Full-width-athalf-maximum (FWHM) for the initial and final spectra are $303 \mathrm{pm}$ and $506 \mathrm{pm}$, respectively.

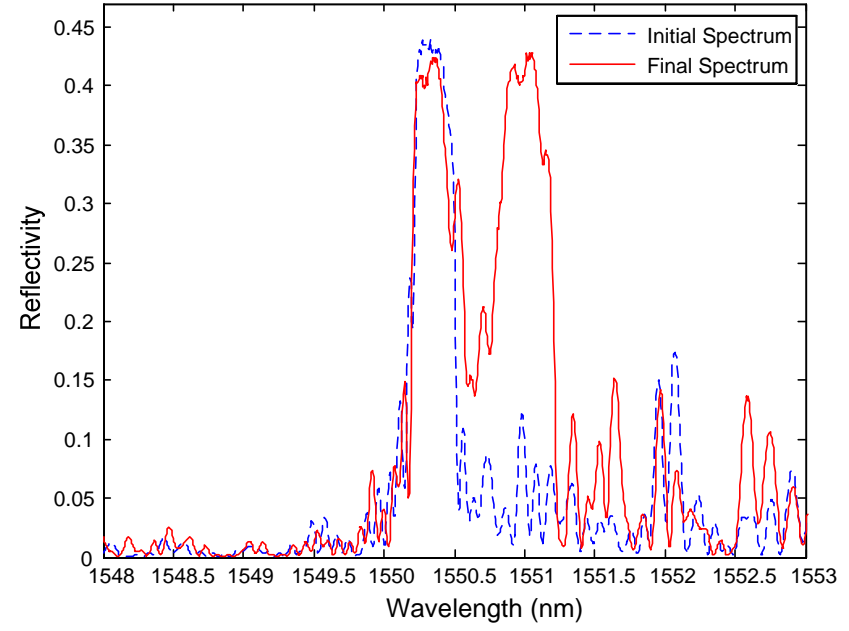

Fig. 3 Initial and micromachined FBGs reflection spectra at a pulse intensity of $1.45 \times 10^{14} \mathrm{~W} / \mathrm{cm}^{2}$

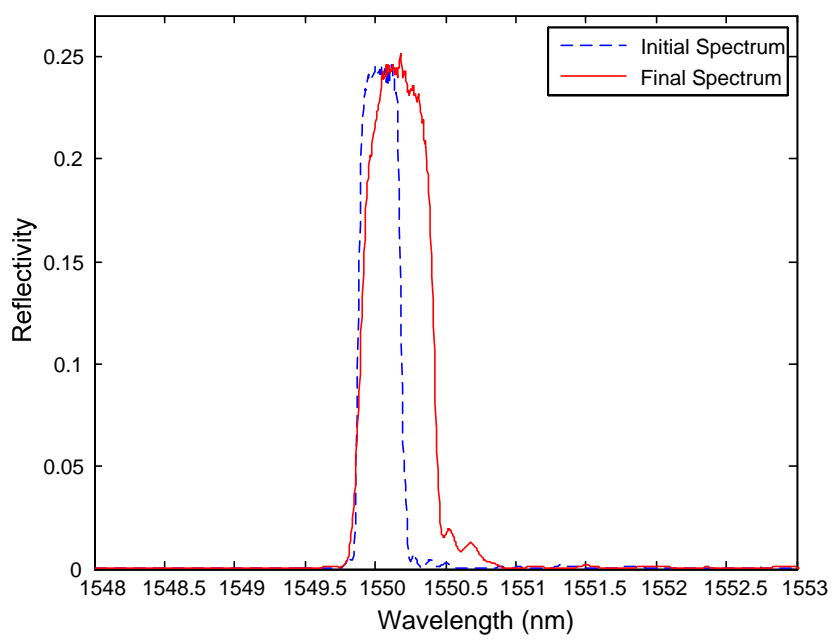

Fig. 4 Initial and micromachined FBGs reflection spectra at a pulse intensity of $9.06 \times 10^{13} \mathrm{~W} / \mathrm{cm}^{2}$

\subsection{Discussions: Optical Response}

According to the experimental results in Fig. 2, 3 and 4, three different scenarios occurred in the interaction of femtosecond laser pulses with FBGs. The modifications are directly attributed to the change in the index of refraction of the FBGs. In the interaction of femtosecond pulses with silica the index of refraction is increased in the exposed areas [9]. The change in the index refraction is a function of pulse intensity; higher pulse intensities result in larger index changes. In Fig. 2, the Bragg wavelength has permanently shifted to a higher wavelength, as the whole length of grating is exposed to laser pulses. However, in Fig. 3, the micromaching was conducted on half of the length of grating, so the initial Bragg wavelength is preserved and another one was generated at a higher wavelength. In Fig. 4, two wavelengths are available. Due to the lower laser pulse intensity, the Bragg peaks spacing is small and are seen as one peak with a larger bandwidth.

In addition to the above-mentioned modifications of the spectral response, the reduction of the sharpness (in Fig. 2, Fig. 3, and Fig. 4), increase of the out-of-band background level (in Fig. 3 and Fig. 4), and grating strength reduction (in Fig. 2) are observed. These are attributed to the phase shifts resulted from the induced perturbations [18].

The modifications of the index of refraction obtained in these experiments are comparable to the results reported in literature [19]. By tuning the laser parameters and characterizing the process, the optical performance of the FBGs can be selectively customized.

\section{Sensing Performance}

In addition to the changes in the optical performance of the FBGs, the femtosecond laser pulses caused the cladding surface to ablate forming periodic micro-grooves.

Fig. 5 shows the structures fabricated on a fiber with the pitch of $50 \mu \mathrm{m}$ at a laser average power of $40 \mathrm{~mW}$ and laser speed of $100 \mu \mathrm{m} / \mathrm{s}$. The width (w) and the depth $(h)$ of the structures are $22 \mu \mathrm{m}$ and $32 \mu \mathrm{m}$, corresponding to 220 pulses per spot on the center line at a laser speed of $100 \mu \mathrm{m}$. 


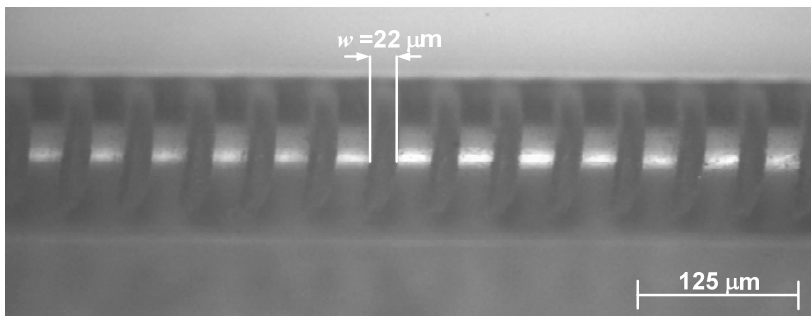

Fig. 5 Periodic microgrooves fabricated on the fiber with laser average power of $40 \mathrm{~mW}$ (pulse energy of $40 \mu \mathrm{J}$ ) and laser speed of $100 \mu \mathrm{m} / \mathrm{s}$

In optical fibers, the effective mode index of refraction $\left(n_{\text {eff }}\right)$ is a function of the indices of refraction of core $\left(n_{\text {core }}\right)$, cladding $\left(n_{\text {clad }}\right)$ and the surrounding medium [20]. Any change in these values may cause $n_{\text {eff }}$ to modify. If an FBG which has had the cladding selectively removed using the laser micromachining process is moved from one medium to another with different refractive index, the effective mode index of refraction and as a result the Bragg wavelength are changed. The effective parameter determining the refractive index of liquids is concentration. To investigate these effects, the micromachined FBG with double resonance wavelength was placed in liquid solutions of different concentrations and the optical responses were obtained. Fig. 6 shows the reflection spectra of the FBG at different temperatures in ethanol and $2.6 \%$ solution of Polyvinyl Butyral (PVB) in ethanol.

According to this figure, while the first resonance wavelength $\left(\lambda_{B_{1}}\right)$ is dependent on both concentration and temperature, the second resonance wavelength $\left(\lambda_{B_{2}}\right)$ is mainly a function of temperature and its sensitivity to the concentration of the surrounding liquid is much lower. Fig. 7 shows the change in the Bragg wavelengths spacing ( $\Phi=\lambda_{B_{2}}-\lambda_{B_{1}}$ ), associated with the resonance wavelengths in Fig. 6, when the sensor is immersed in ethanol and PVB solutions in ethanol. According to this figure, the Bragg wavelengths spacing decreases with increasing the concentration.

As seen in the experimental results, the sensitivity of the micromachined FBGs to liquid concentration was increased as a result of the fabrication of corrugated structures on the cladding. When the corrugated structures are fabricated, the cladding effective thickness is decreased and as a result the effects of the refractive index of the surrounding liquid become more significant in the coreconfined propagation modes of the optical fiber. The Bragg condition is a function of $n_{\text {eff }}$ and as a result, a Bragg wavelength shift occurs when a corrugated FBG is placed in a liquid with different refractive index. According to (1), in an FBG with two resonance wavelengths, e.g., Fig. 6, the wavelength spacing ( $\Phi$ ) is

$$
\Phi=\lambda_{B_{2}}-\lambda_{B_{1}}=2 \Delta n_{\text {eff }} \Lambda
$$

In this case, increasing the concentration and hence the index of refraction of the surrounding medium causes $\Delta n_{\text {eff }}$ to decrease accordingly which reduces $\Phi$.

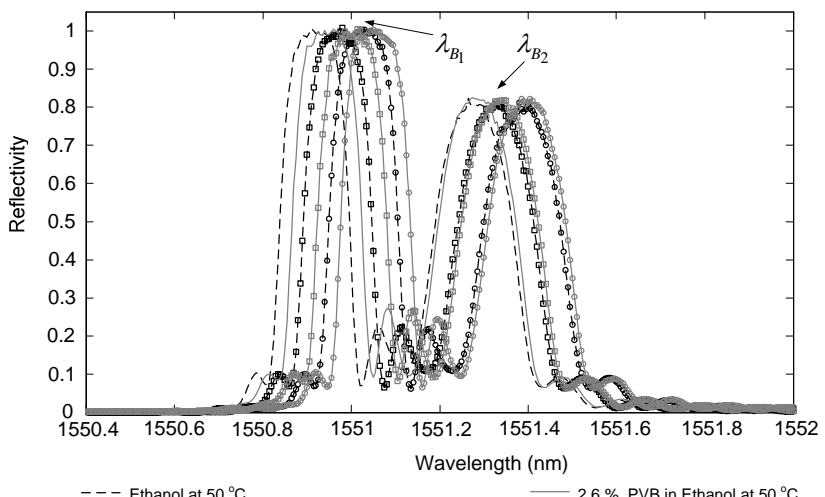

--- Ethanol at $50^{\circ} \mathrm{C}$
- - Ethanol at $55^{\circ} \mathrm{C}$
$-\theta-$ Ethanol at $60^{\circ} \mathrm{C}$

$-2.6 \%$, PVB in Ethanol at $50{ }^{\circ} \mathrm{C}$
$-2.6 \%$, PVB in Ethanol at $55{ }^{\circ} \mathrm{C}$
$-2.6 \%$, PVB in Ethanol at $60^{\circ} \mathrm{C}$

Fig. 6 Reflection spectra of the micromachined FBG sample with dual resonance wavelengths at different temperatures in ethanol and PVB solution in ethanol

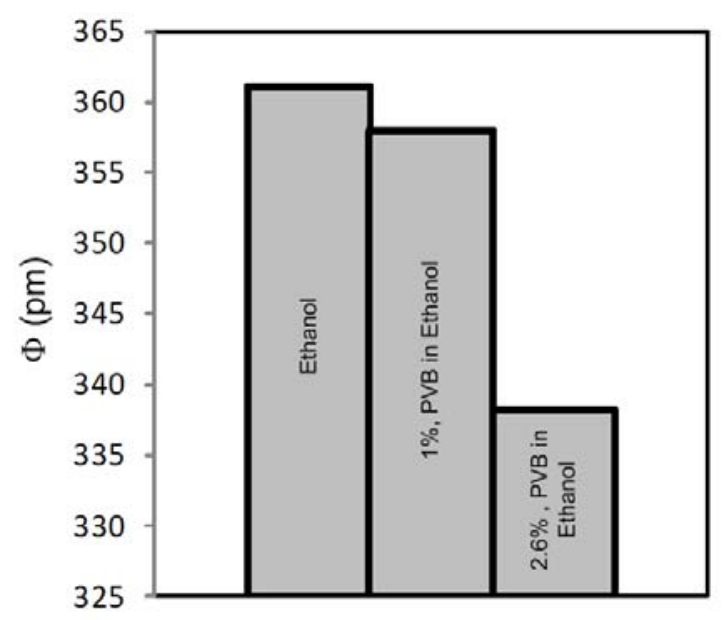

Fig. 7-Average Bragg wavelengths spacing ( $\Phi$ ) for different liquid concentrations

In addition, according to the experimental results, the FBGs are still sensitive to temperature which means that, in contrast to previous FBG sensors reported in the literature (e.g. [11-12]), a single sensor of the type developed here using selective laser micromachining can be used for simultaneous measurement of both temperature and concentration of liquids.

\section{Conclusions}

In this paper we reported the femtosecond laser micromachining of fiber Bragg gratings to modify their performance. Different scenarios were investigated in terms of the laser processing parameters and laser pulse intensity. Permanent change of the Bragg wavelength, reflection peak splitting and bandwidth increase are among the effects that happened in the micromachined FBGs. In addition, the sensing performance of the micro-machined FBGs was increased by the inscription of periodic micro-grooves along the fiber axis. The micromachined fibers were successfully used in the simultaneous measurement of concentration and temperature of liquids.

\section{Acknowledgments}

The authors are pleased to express their appreciation for the cooperation of Dr. Andrew Pinkerton, Dr. Amin Abdol- 
vand and Dr. David Whitehead at the Laser Processing Research Centre (LPRC) of the University of Manchester, and the financial support from National Sciences and Engineering Research Council (NSERC) of Canada and Ministry of Research and Innovation of Ontario.

\section{References}

[1] A. Othonos and K. Kalli: "Fiber Bragg Gratings" (Artech House, Boston, 1999) p. 301.

[2] C. B. Schaffer, A. Brodeur, and E. Mazur: J. Meas. Sci. Technol., 12, (2001) 1784.

[3] C. B. Schaffer, A. Brodeur, J. F. García, and E. Mazur: Optics Letters, 26, (2001) 93.

[4] J. Kruger and W. Kautek: "Advances in Polymer Science” ed. by Lippert T. (Springer, Berlin / Heidelberg, 2004) 168 p. 247.

[5] X. Liu, D. Du, and G. Mourou: IEEE J. Quantum Electronics, 33, (1997) 1706.

[6] M. Lenzner, J. Kruger, S. Sartania, Z. Cheng, C. Spielmann, G. Mourou, W. Kautek, and F. Krausz: Physical Review Letters, 80, (1998) 4076.

[7] Chan J W, Huser T, Risbud S and Krol D M 2001 Structural changes in fused silica after exposure to focused femtosecond laser pulses Optics Letters 26 1726-1728.

[8] B. Poumellec, L. Sudrie, M. Franco, B. Prade, and B. Mysyrowicz: Optics Express, 11, (2003) 1070.

[9] R. R. Gattass and E. Mazur: Nature Photonics, 2, (2008) 219.

[10]D. Grobnic, S. J. Mihailov, and C. W. Smelser: J. Lightwave Technology, 25, (2007) 1996.

[11]S. J. Mihailov, C. W. Smelser, D. Grobnic, R. b. Walker, P. Lu, H. Ding, and J. Unruh: J. Lightwave Technology, 22, (2004) 94.

[12]A. Martinez, M. Dubov, I. Khrushchev, and I. Bennion: Electronics Letters, 40, (2004) 1170

[13]J. Thomas, E. Wikszak, T. Clausnitzer, U. Fuchs, U. Zeitner, S. Nolte, and A. Tunnermann: J. Applied Physics A, 86, (2007) 153.

[14]C. Zhan C, J. Lee, S. Yin, P. Ruffin, and J. Grant: J. Applied Physics, 101, (2007) 053110.

[15]E. Wikszak, J. Thomas, S. Klingebiel, B. Ortaç, J. Limpert, S. Nolte, and A. Tunnermann: Optics Letters, 32, (2007) 2756.

[16]T. Erdogan: J. Lightwave Technology, 15, (1997) 1277.

[17]F. Durr, H. G. Limberger, R. P. Salathe, F. Hindle, M. Douay, E. Fertwin, and C. Przygodzki: Applied Physics Letters, 84, (2004) 4983.

[18]R. Feced, and M. N. Zervas: J. Lightwave Technol., 18, (200), 90.

[19]D. M. Krol: J. of Noncrystalline Solids, 354, (2008) 416.

[20] J. A. Buck: "Fundamentals of Optical Fibers” (John Wiley \& Sons, Hoboken, 2004) Chapter 3.

(Received: June 16, 2008, Accepted: March 10, 2009) 